\title{
Approche iconoclaste d'Enoch Powell au ministère de la Santé (1960-1963) : entre liberté économique et puissance étatique?
}

Enoch Powell's Iconoclastic Approach to the NHS (1960-1963): Promoting

Economic Freedom and State Power?

\section{Stéphane Porion}

\section{OpenEdition \\ Journals}

Édition électronique

URL : http://journals.openedition.org/rfcb/4373

DOI : $10.4000 /$ rfcb.4373

ISSN : 2429-4373

Éditeur

CRECIB - Centre de recherche et d'études en civilisation britannique

Référence électronique

Stéphane Porion, «Approche iconoclaste d'Enoch Powell au ministère de la Santé (1960-1963) : entre liberté économique et puissance étatique? ", Revue Française de Civilisation Britannique [En ligne], XXIV-3 | 2019, mis en ligne le 30 août 2019, consulté le 04 septembre 2019. URL : http:// journals.openedition.org/rfcb/4373; DOI : 10.4000/rfcb.4373

Ce document a été généré automatiquement le 4 septembre 2019

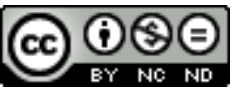

Revue française de civilisation britannique est mis à disposition selon les termes de la licence Creative Commons Attribution - Pas d'Utilisation Commerciale - Pas de Modification 4.0 International. 


\title{
Approche iconoclaste d'Enoch Powell au ministère de la Santé (1960-1963) : entre liberté économique et puissance étatique?
}

\author{
Enoch Powell's Iconoclastic Approach to the NHS (1960-1963): Promoting \\ Economic Freedom and State Power?
}

Stéphane Porion

\section{Introduction}

1 Aneurin Bevan, véritable architecte du National Health Service (NHS), déclarait avec force après sa démission du ministère de la Santé en 1950 : «A free health service is a triumphant example of the superiority of collective action and public initiative applied to a segment of society where commercial principles are seen at their worst $»^{1}$. À ses yeux, le principe collectiviste avait permis à tous les Britanniques malades d'avoir accès au soin gratuitement dans une société qu'il jugeait ainsi "civilisée», aux antipodes de «l'hédonisme d'une société capitaliste $»^{2}$. Le NHS avait rapidement remporté l'adhésion et l'affection des Britanniques dans la mesure où il assurait un système de soins universel, global et relativement gratuit depuis sa mise en place en 1948. Cependant, il s'est avéré rapidement que ce système de santé, aussi populaire qu'il fut, ne parvint pas à résoudre trois problèmes fondamentaux d'ordre administratif, politique et économique, qui devinrent des préoccupations grandissantes pour les différents ministres de la Santé. La hausse de la demande de soins accentuait le problème des ressources suffisantes et le financement du NHS. Les méthodes de planification centralisée étaient encore peu développées et la structure même du NHS posait problème; ce que reconnaissaient volontiers les professionnels du secteur au début des années 1960 : « The tripartite system discouraged rather than encouraged cooperation and only in the 1960s did the Ministry of Health start seriously to develop the skills by which relative merits of centralised 
planning (as opposed to those of the market and local initiative) could be truly tested $»^{3}$. Même si un consensus perdurait avec l'idée de " universalising the best », le NHS originel conçu par Bevan semblait incapable d'apporter une solution à ces multiples défis.

2 Entre 1951 et 1964, sept ministres conservateurs de la Santé se succédèrent, ne bénéficiant du statut de ministre du Cabinet que pendant une période de deux ans (de 1962 à 1964). Bien que la plupart d'entre eux aient souvent été de moindre envergure, Enoch Powell semble faire exception aux yeux des historiens du NHS ${ }^{4}$. Par ailleurs, la nomination de l'ancien haut fonctionnaire au ministère de l'Économie et des finances ( former Treasury official), Sir Bruce Fraser, au poste de secrétaire permanent du Ministère de la Santé (Permanent Secretary), ainsi que celle de Sir Gorge Godber en tant que directeur général de la Santé (Chief Medical Officer) au début des années soixante, ont conduit à un leadership administratif fort au côté de Powell dans «un ministère revitalisé $»^{5}$. Selon Nicholas Timmins, « it was a triumvirate of some note who took the NHS into the $1960 \mathrm{~s} »^{6}$.

Non seulement Powell reconnut lui-même que devenir ministre de la Santé lui permit de se confronter à un nouveau défi intellectuel, mais il conçut également son rôle à ce ministère de manière originale, désireux de réaliser un ou deux projets de grande envergure afin de laisser sa marque dans ce secteur ${ }^{7}$. Il lança notamment un vaste plan ambitieux de construction et de modernisation des hôpitaux en Grande-Bretagne, et comptait révolutionner la façon dont étaient traités les problèmes de santé mentale, en améliorant les infrastructures et en développant les soins et services d'aide à domicile, hors des instituts spécialisés. Powell se rappelait très bien que lorsque Macmillan devint Premier ministre en 1957, le Parti conservateur s'était engagé à augmenter à la fois les dépenses de santé au vu de l'évolution démographique du pays, ainsi que les dépenses d'investissement, dont une partie serait consacrée à la modernisation des hôpitaux ${ }^{8}$. Il se trouvait donc dans une situation paradoxale que l'un de ses biographes (Robert Shepherd) a qualifiée de « contorted logic " ${ }^{9}:$ il espérait œuvrer pour la modernisation du NHS, tout en rationalisant les dépenses de son ministère - objectif qu'il connaissait bien, ayant été secrétaire financier du ministre de l'Économie et des Finances en 1957-1958 et démissionné à cause d'un manque de volonté de la part du Cabinet de Macmillan d'enrayer la hausse des dépenses publiques, génératrice à ses yeux d'inflation ${ }^{10}$.

Jusqu'en 1960, malgré la pression des ministres de l'Économie et des Finances, ceux de la Santé furent en effet peu enclins à maîtriser leurs dépenses toujours en constante augmentation. Charles Webster estime que cette situation changea en juillet 1960 avec la nomination de Powell au ministère de la Santé : «[His] appointment meant that, for the first time, the Treasury and the Ministry of Health were agreed in their philosophy towards the welfare state in general $\aleph^{11}$. Powell reconnut d'ailleurs lui-même que sa position ne divergea jamais de celle du chancelier de l'Echiquier pendant ses années passées à la Santé12. Toutefois, il justifiait ce besoin d'humaniser le NHS :

Let us not make the mistake of supposing that this unity of the National Health Service can be produced just by clever organisation, by efficient administration, or even by directives, however well composed, from the ministry of Health. The true unity of the National Health Service emerges from its unity in the service of the patient. That is its true, fundamental, psychological unity, and of that we must never lose sight. This has been the impelling force behind all that has been and is being done for what is sometimes called the humanisation of the National Health Service. The Service has been intensely conscious of this duty laid upon it in recent years. (...) [T] he service is alive, as it must be alive, to its ultimate dedication to the 
patient. (...) [T] $]$ he Service is given its meaning, is given its justification and is given its unity by one thing only, its duty to the individual suffering man or woman ${ }^{13}$. livres par an, son personnel avait le devoir d'honorer la mission qui lui avait été confiée ; en d'autres termes œuvrer pour le bien-être, la dignité et le respect du patient dans son traitement ${ }^{14}$. Ainsi, Powell, véritable chantre d'idées libérales, soucieux de défendre les sacrosaints principes de liberté et de choix individuels, se trouvait à la tête d'un ministère à moderniser. Malgré ce positionnement clairement établi depuis son expérience au ministère de l'Économie et des Finances, Powell décida de garder un service de Santé public tout en ne reniant pas son credo libéral; ce qui en fit un ministre de la Santé iconoclaste.

Certaines biographies sur Powell ont analysé ses années passées au ministère de la Santé, tentant qu'expliquer sa stratégie paradoxale d'humanisation et de rationalisation du NHS. Par exemple, Robert Shepherd et Simon Heffer se sont principalement concentrés sur le plan de modernisation des hôpitaux, les problèmes du service hospitalier de santé mentale et du budget difficilement maîtrisable de ce ministère, et la crise avec les infirmières sur la question des salaires ${ }^{15}$. D'autres études sur le NHS mentionnent à quelques reprises le nom de Powell : pour Ian Holliday, le plan de construction des hôpitaux mis en place par Powell a permis de révéler des «faiblesses structurelles » du $\mathrm{NHS}^{16}$. Brian Watkin estime aussi que Powell avait déjà diagnostiqué les problèmes affectant le personnel médical au sein des hôpitaux et des maternités bien avant la publication de l'ouvrage de Gerda Cohen en 1964 (What's Wrong with Hospitals?) ${ }^{17}$. Selon Virginia Berridge qui partage le point de vue d'Harriet Jones, Powell était un bon exemple du dilemme auquel était confronté un ministre de la Santé : «the tension between what was ideologically and economically desirable and what was politically feasible $»^{18}$. Quant à Charles Webster, il explique pourquoi Powell fut le dernier ministre de la Santé à souscrire à l'opinion que le NHS était un secteur où l'on pouvait réellement diminuer les dépenses publiques ${ }^{19}$. Enfin mentionnons l'article de Tony Cutler publié en 2011, qui a montré que Powell avait suivi une logique libérale cohérente dans la mise en place de son plan de construction et de modernisation des hôpitaux malgré la dimension de planification du projet; pour cet auteur, ce fut surtout la stratégie de Powell qui posa problème ${ }^{20}$.

7 Cet article vise ainsi à compléter les débats historiographiques et mettre en lumière d'autres enjeux que ceux mentionnés précédemment, qui ont également marqué les années Powell au ministère de la Santé. Il s'intéressera entre autres aux problématiques de l'achat de médicaments et de son impact financier sur le NHS, mais aussi à la question du tabagisme qui s'inscrit plutôt dans le domaine de santé publique. Ces enjeux seront abordés autour d'un autre dilemme que Powell dut gérer que celui d'humaniser/ rationnaliser le NHS : jusqu'à quel point était-il possible de défendre les idées de liberté et de choix dans un système public de santé où la puissance étatique était force motrice? Dans leur ouvrage intitulé Welfare Policy under the Conservatives (1951-1964), Paul Bridgen et Rodney Lowe répertorient les archives nationales portant sur les politiques sociales des treize années au pouvoir des Conservateurs et indiquent, dans leur section sur Powell au ministère de la Santé, que les sources primaires permettent essentiellement de mettre en lumière l'élaboration et la mise en place de son plan de modernisation des hôpitaux, ainsi que la manière dont il a géré le budget d'un ministère dispendieux ${ }^{21}$. En s'appuyant sur ces mêmes sources primaires, complétées par les archives personnelles de Powell 
détenues au Churchill Archives Centre à Cambridge, l'étude abordera ainsi les points suivants qui n'ont été encore à ce jour que partiellement analysés: comment Powell concevait la nature et la gestion du NHS, puis comment le ministre géra les questions de coût des médicaments, de la publicité sur le tabac et de la fluoration de l'eau.

\section{Approche powellienne du NHS}

8 Au tournant des années 1960, deux questions apparaissaient problématiques pour les Conservateurs et méritaient réflexion: fallait-il qu'ils défendent la philosophie de l'ÉtatProvidence mis en place par Clement Attlee impliquant des services sociaux universels assurés par la puissance étatique, ou devaient-ils promouvoir à la fois l'idée de sélectivité des aides sociales publiques à destination des plus fragiles de la société et les canaux privés d'assurances pour ceux qui en avaient les moyens? De plus, la question de l'augmentation des dépenses publiques visant à assurer un niveau de couverture sociale universelle et globale avait suscité de vifs débats au sein du Cabinet de Macmillan en 1957 et janvier 1958, menant à la démission des trois ministres de l'Économie et des Finances, dont Enoch Powell. Ce fut dans ce climat qu'émergèrent des idées plutôt radicales de la part de certains Conservateurs qui contestaient entre autres une gestion étatique du NHS, lorsque Powell devint ministre. Citons-en quelques- unes qui allaient s'inscrire en porteà-faux avec la vision powellienne du NHS. Par exemple, dans un article publié dans Crossbow (revue du Bow Group) en 1959 et intitulé « Reshaping Welfare », Brendon Sewill défendit l'idée selon laquelle, à cause de la pression exercée pour contenir les dépenses publiques, il devrait y avoir une réduction d'impôts pour ceux qui souscriraient une assurance de santé privée, « equivalent to the per capita cost of the NHS $»^{22}$.

Par ailleurs, D. S. Lees de Keele publia un article en 1961 par le biais de l'IEA (Institute of Economic Affairs), intitulé Health through Choice, et affirma: "Medical care should increasingly be based on consumer choice and supplied through the market $»^{23}$. Il estimait en effet, qu'à cause du NHS, les décisions politiques supplantaient les choix des individus, et que par voie de conséquences, les dépenses allouées au NHS étaient alors bien supérieures à ce qu'elles auraient été si le système de santé avait été géré par les forces du marché. Il ajoutait qu'un service étatisé et uniforme, laissé aux seules mains et décisions d'un ministre, empêchait le système de s'adapter aux changements et la liberté d'expérimenter. Ce dernier se trouvait aussi déconnecté du terrain et des professionnels de santé. De manière plus controversée, il avançait l'idée selon laquelle le NHS, de par sa nature étatisée, n'avait pas vraiment permis d'améliorer le niveau de santé des Britanniques; seules les classes moyennes tiraient réellement profit du système. Ainsi, à ses yeux, les soins médicaux ne devraient pas être considérés comme une forme d'investissement, mais comme un bien de consommation avec un prix à payer. Selon Timothy Raison, «Right up to 1987 official Conservative party policy shied away from Lee's approach, feeling that the NHS was too popular an institution to replace or even seriously question $»^{24}$.

10 Plus généralement, au tournant des années 1960, l'IEA contestait la philosophie de l'ÉtatProvidence et développait le principe selon lequel il devrait y avoir plus de choix et de liberté. Deux autres Conservateurs relayaient le même message. Keith Joseph pensait que c'était une erreur d'attribuer à l'État le rôle permanent de garantir à tous une couverture sociale élémentaire. Quant à Geoffrey Howe, il partageait la vision de Lees et estimait que 
la Santé devrait être gérée par le secteur privé afin d'éviter ce qui allait être considéré par les partisans du thatchérisme comme « la culture de l'assistanat ${ }^{25}$.

11 Au début des années 1950, Ian Macleod, alors ministre conservateur de la Santé, avait accepté, selon Charles Webster, la philosophie travailliste du NHS : « Macleod effectively purged residual rancour towards Bevan's health service in the Conservative Party $»^{26}$. Il explique que Macleod voyait le NHS comme l'aboutissement logique d'une tradition respectable ancrée dans la philosophie sociale disraélienne. Macleod avait indéniablement été influencé par son appartenance au "One Nation Group ». Il publia d'ailleurs en 1952 avec Powell, lui-même membre de ce "dining group", une étude intitulée Social Services: Needs and Means qui reflétait cette approche disraélienne de l'ÉtatProvidence. Force est de constater que même si Powell s'inscrivait dans cette même approche au début des années 1950, son expérience en tant que secrétaire d'État au ministère de l'Économie et des Finances (Financial Secretary) en 1957-1958 changea entre autres sa vision du financement et du fonctionnement du NHS. Ce même auteur ajoute également : « Powell soon returned from the wilderness to become minister of Health, in which capacity he embarked on an exercise of self-vindication by once again applying his retrenchment philosophy to the health service. The confrontation with the nurses was merely one manifestation of this effort, [as well as] further characteristic interventions concerning health-service charges and the NHS Contribution $»^{27}$. Malgré une posture consistant à être perçu comme un ministre de l'Économie sous couverture, Powell décida de garder la structure publique du NHS, tout en ne perdant pas de vue les problématiques de liberté et de choix individuels.

Dans son ouvrage Medicine and Politics, publié en 1966, Powell expliquait ainsi que les soins médicaux pouvant être, par nature, des besoins aussi vitaux que se nourrir ou se loger, ils relevaient donc d'une gestion étatique ${ }^{28}$. Plus généralement, il soulevait à nouveau au début des années 1960 la question centrale qui devait guider tout choix politique :

At the centre of politics today lies, as never before, the question of what Government should do, or abstain from doing, to control, determine, regulate or alter the behaviour of its subjects in the economic field. There have lately been signs that Conservative leaders either themselves lack clearly defined ideas about the limits and conditions of such Government action. (...) Where matters are not defined by authority, the individual believer is at liberty to use his own judgment ${ }^{29}$.

13 Selon Powell, il existait deux sortes de critères : "des critères économiques » et des "critères non économiques" de "nature philanthropique ${ }^{30}$. Ces critères retenus par Powell font sens au regard des analyses sur le concept de «biens publics » développées par Paul Samuelson ${ }^{31}$, ainsi que de celles sur les soins médicaux («medi-care ») menées par Kenneth Arrow $^{32}$. Parmi les critères non économiques, Powell y plaçait, par exemple, la gestion publique de la santé: "In instituting a universal free medical service, a Government does not act on economic criteria. It acts on the ground that it is a 'good thing' for its subjects to be cured of their ailments, if humanly possible $»^{33}$. Sa position idéologique reflétait, dans une certaine mesure, celle d'Adam Smith développée dans The Wealth of Nations (1776), laquelle avait déterminé des secteurs d'activité compatibles avec une ingérence étatique servant au mieux l'intérêt individuel qui se fondait, dans ce cas précis, sur l'intérêt collectif. Toutefois, la pensée de Powell faisait aussi précisément écho à la philosophie d'Hayek et plus particulièrement à « son ordre spontané » :

One class of decisions looks to areas where no spontaneous process can operate.

The decisions to organize a free health service can only be a decision of 
government. The free choice of individuals will not automatically build [a hospital]

34.

Powell concédait toutefois que gérer un service public de santé entraînait des conséquences économiques, et qu'il fallait ainsi laisser un choix viable aux individus s'ils décidaient d'avoir recours à des soins dispensés par le secteur privé. Powell prenait également en compte la dimension humaine du débat en intégrant les «critères nonéconomiques $»^{35}$; ce qui motiva sûrement sa volonté de développer les hôpitaux et d'améliorer les traitements pour les patients atteints d'une maladie mentale. Il ne faudrait pas, cependant, en déduire que Powell était un fervent défenseur de la planification et un inconditionnel converti aux bienfaits des nationalisations. Considérant le critère humain comme prioritaire, il expliquait dans quel état d'esprit il avait envisagé la planification de la construction et de la modernisation des hôpitaux :

I therefore had to form patterns and make genuine plans - in the proper sense of a plan, not a plan about what other people would do but a plan about what you yourself or your successors would do. For good or ill, therefore, the Minister was in a position to mould the pattern of the hospital service, the pattern of the mental health service and the pattern of the community or health and welfare services ${ }^{36}$.

Après son passage au ministère de la Santé, Powell fit en 1966 un bilan de son action et dénonça de nouveau le problème de la gratuité du service, qui entrainait de facto à ses yeux " une demande illimitée », laquelle était toutefois circonscrite par une offre limitée : "Whatever assumptions one makes about generosity, (...) it is that point of conflict, between the potential of the unlimited demand and the supply, which has to be somehow cut down to meet it $\aleph^{37}$. Toutefois, Powell ne soutenait pas la disparition du NHS au profit d'un système de santé privé car plus le consommateur aurait de choix, plus il dépenserait pour sa santé. Ainsi, les patients pourraient décider de consulter plusieurs médecins, échappant au système public unique, censé garantir la gratuité des soins à tous les individus et l'uniformisation de leur prise en charge ${ }^{38}$. Si les médecins désiraient une plus grande liberté - que ce système public ne pouvait leur octroyer -, ils devraient chercher eux-mêmes d'autres sources de financements que celles de l'État ${ }^{39}$. Selon Nicholas Barr, même si Powell avait des inclinations libérales bien marquées, il avait fait le bon choix de conserver un système de santé public :

It was acknowledged that a centralized NHS was able to deliver a comparable standard of health care more cost-effectively than the systems of private and social insurance practised in the USA and continental Europe. Policy makers, in short, had grasped the later economic wisdom that governments could do things which private markets for technical reasons either would not do at all, or would do ineffectively ${ }^{40}$.

Malgré la nature centralisée et étatisée du ministère de la Santé, Powell décida dès le départ d'inclure tous les présidents des agences régionales de santé dans les débats et les prises de décision, en les conviant une fois par mois à une réunion à son ministère ${ }^{41}$. Il déclarait en effet qu'ils se comportaient comme une équipe, arrivant à des conclusions concertées en échangeant et en réfléchissant ensemble ${ }^{42}$. Selon lui, sa méthode permit d'instaurer un mode consensuel efficace au sein de son ministère: "I never lost by seeking agreement round the table to alterations in financial allocation, to changes of policy, and to planning decisions, even though technically the decisions were mine $»^{43}$. Powell opta sûrement pour un tel mode de fonctionnement afin de contourner le système bureaucratique dont la nature pouvait rapidement s'avérer problématique à ses yeux. ${ }^{44}$. Selon Neil Small, « Powell combined an appreciation of Realpolitik with a theoretical and ethical appreciation of something he defined as public duty $»^{45}$. Alors que l'action de 
Powell au ministère de la Santé déclencha de vives réactions parmi certains professionnels du milieu ${ }^{46}$, il laissa plutôt une image positive, vu comme " a man of principle, determined, enthusiastic, who has the courage to act according to his convictions $»^{47}$.

17 Powell dut naturellement gérer la question des finances. Selon lui, " the unnerving discovery every Minister of Health makes at or near the outset of his term of office is that the only subject he is ever destined to discuss with the medical profession is money $»^{48}$. Il dut en effet veiller constamment à la maitrise des dépenses de santé, ce qui lui valut des conflits avec certains collègues au sujet de la gratuité des médicaments pour les patients passant par le système privé de santé, ainsi qu'avec certains membres de la profession médicale, tels que les infirmières, par rapport à des revendications salariales en 1962. Powell dut faire face à "la litanie perpétuelle et assourdissante de plaintes qui [émanaient] jour et nuit de toutes parts $\aleph^{49}$. Il devait assumer la décision du gouvernement de contenir les hausses de dépenses publiques et de celles d'investissement. Robert Shepherd appela cet objectif " Powell's 'contract' with the Treasury $\aleph^{50}$. Ce dernier avait en effet accepté en 1961 que les dépenses de son ministère n'augmentent que de $2,5 \%$ par an jusqu'en $1965 / 1966^{51}$, ce qui correspondait à l'objectif du Trésor de contrôler le coût de l'évolution des politiques publiques par la mise en place du Public Expenditure Survey Committee $e^{52}$. Ainsi, Powell fut perçu comme agissant encore comme secrétaire financier, avec pour mission de maîtriser les dépenses publiques ${ }^{53}$.

\section{Le coût des médicaments : combat de Powell contre l'industrie pharmaceutique}

18 Dès sa nomination au ministère de la Santé, Enoch Powell reçut une lettre de cadrage du Premier Ministre, soulignant les enjeux importants auxquels le NHS était confronté : " There is really the future of the Service, which way it will go. And then there is the terrible expense that ought in some way be reduced, especially in the form of the drug bill $\aleph^{54}$. Il fut ainsi mandaté pour s'attaquer à la question du coût exorbitant des médicaments qui grevait le budget de son ministère. Les entreprises pharmaceutiques réalisaient en effet d'énormes profits, qu'elles justifiaient par les fonds engagés pour la recherche, la mise au point de ces médicaments et les frais de commercialisation. Enoch Powell analysa ce problème sous l'angle d'une « relation unique entre le fournisseur et le consommateur $"^{55}$ et indiqua que l'achat d'un médicament par le consommateur faisait intervenir trois acteurs différents au sein du NHS - les médecins commandent les médicaments, les patients les consomment, mais le ministre paie ces dépenses : «I pay the bill, but I don't order the goods; and I don't consume them either $» .^{56}$ De plus, les industries pharmaceutiques n'avaient à traiter qu'avec un seul client sur le marché britannique - le NHS. Alors qu'une étude menée aux États-Unis (le rapport Kefauver) venait de révéler que le problème résidait dans le monopole d'approvisionnement en médicaments détenu par les industries pharmaceutiques britanniques, lesquelles pouvaient ainsi librement fixer un prix de vente élevé au ministère de la Santé, puisqu'elles étaient protégées par la loi sur les brevets datant de $1949^{57}$, Powell décida d'agir en $1961^{58}$ déclarant: " The pharmaceutical firms cannot view with indifference the danger of being branded as profiteers (...) making large profits out of the sick $»^{59}$. 

la tétracycline. Les entreprises en possession d'une licence pour la commercialiser en demandaient 90 livres pour mille comprimés, sous prétexte qu'il s'agissait d'un antibiotique efficace couvrant un plus large spectre de maladies infectieuses que la pénicilline. Au début des années soixante, son prix de vente avait baissé d'environ 30\% pour avoisiner les 60 livres. En 1961, une nouvelle entreprise pharmaceutique, DDSA Pharmaceuticals, pouvait commercialiser la tétracycline au prix de 6 livres 10 shillings pour 1000 tablettes, soit dix fois moins que ce qu'en demandait Pfizer ${ }^{60}$. Toutefois, A. M. Brunton, directeur médical du groupe Pfizer, justifiait ainsi ce prix élevé :

As soon as a valuable new drug is discovered and scientific data published for all the world to see, any chemical manufacturer can, if patents are ignored, enter into production of the discoverer's new drug without having incurred any of the risks and expenses involved in the research and development of the compound ${ }^{61}$.

Le rapport Kefauver rappelait que l'action de Pfizer était justifiée par une décision antérieure relative au système des brevets déposés : il fallait encourager l'esprit inventif au détriment de la concurrence. Ainsi, les laboratoires disposaient du monopole pour fixer leur prix de vente sans avoir à craindre qu'une autre entreprise ne commercialise leur médicament à un prix inférieur ${ }^{62}$.

21 Enoch Powell, «l'apôtre du secteur privé " ${ }^{63}$, allait modifier les choses en profondeur en poussant l'État à injecter de la concurrence dans l'optique de faire baisser les prix. Selon lui, « the government still possesses statutory power to control the prices of drugs. It is a power about as useful, for practical purposes, as a hydrogen bomb in the Vietnam war ${ }^{64}$. Lors d'un discours prononcé devant l'Association des Industries Pharmaceutiques Britanniques le 27 avril 1961, il affirma que le système de libre concurrence et de liberté des prix procure de plus grands bénéfices à tous les catégories de la société que tout autre système alternatif ${ }^{65}$. Powell rappela que, sans concurrence ni liberté des prix, le système actuel pouvait entraîner de sérieux blocages de la part des deux parties (fournisseurs et consommateurs) qui le mèneraient à sa perte :

My weakness is that the Health Service must have the drugs you supply: where a patient's life may depend on having a particular proprietary drug, I cannot refuse on financial grounds to let his doctor order it for him. Your weakness is that virtually your only home market for your ethical drugs is the National Health Service: if I limit the scope or the profitability of that market, you have no alternative outlet. This surely creates for each party not merely a moral duty but a practical necessity, in sheer self-interest, to be fair and reasonable in dealing with the other (...) the one side seeking a good bargain in its vast expenditure, the other looking to earn a profit in fair competition, and both believing that the public good is served by their respective efforts ${ }^{66}$.

Enoch Powell eut ainsi recours à l'article 46 de la loi sur les brevets d'invention de 1949, conçue par Sir Stafford Cripps - alors ministre travailliste et avocat spécialisé en droit des brevets. Celui-ci permettait au gouvernement, en temps de guerre, s'il avait besoin d'un produit breveté, de se le faire fournir par une entreprise sans permis de commercialisation à condition de reverser des royalties à l'inventeur du brevet ${ }^{67}$. C'est ainsi que, le 17 mai 1961, Powell accorda le droit à des entreprises sans permis de commercialisation de fournir au gouvernement, même en temps de paix, trois antibiotiques: la tétracycline, le chloramphénicol et le chlorothiazide ${ }^{68}$. Son action se limita aux hôpitaux qui représentaient 14 millions de livres du total annuel du coût des médicaments, lequel s'élevait quant à lui à 110 millions de livres ${ }^{69}$. Powell conclut ainsi un accord commercial en 1962 avec la DDSA pour qu'elle approvisionne les hôpitaux en 
tétracycline pour un montant de 240000 livres $^{70}$. Quant au groupe Pfizer, il attaqua la décision de Powell en justice mais perdit devant la Chambre des Lords en novembre 1964 ${ }^{71}$. Powell remarqua, en 1965, que le gouvernement n'avait plus besoin d'avoir recours à la loi de 1949 et ne renouvela pas ses contrats commerciaux avec les entreprises sans permis de commercialisation, puisque les entreprises brevetées offraient désormais des prix de vente que le ministère jugeait « acceptables $»^{72}$. Ainsi, Powell avait réussi à contraindre ce marché à s'ouvrir à la concurrence pour faire baisser les prix des médicaments en n'hésitant pas à utiliser les pouvoirs de l'État.

\section{Interdire la publicité des cigarettes?}

La Commission des affaires sociales s'était penchée en 1959 sur la corrélation établie entre la consommation de cigarettes et le cancer des poumons. Le ministre de la Santé, Derek Walker-Smith, observait seulement que la consommation de tabac et le nombre de décès provoqués par le cancer des poumons avaient tous deux continué à augmenter depuis que le gouvernement avait décidé d'adopter une politique de transparence: les faits étaient connus par le grand public qui était amené à en tirer ses propres conclusions. Il préconisait de camper sur cette position ${ }^{73}$. Virginia Berridge estime que le tournant des années soixante fut marqué par un véritable bouleversement dans la politique du gouvernement en matière de campagne d'information de santé préventive (health education) :

The late 1950s and early 1960s saw a reorientation of that wartime stance on the part of government: citizens who would act responsibly if given 'the facts' were replaced by consumers of harmful substances who needed to be persuaded about risk $^{74}$.

Ce profond changement dans la politique gouvernementale visait à réconcilier deux positions antinomiques : laisser l'individu libre et responsable de ses actes ou lui imposer des choix de vie. Les comptes rendus des réunions du Cabinet soulignent bien ce dilemme dans la stratégie du gouvernement. Alors qu'il était question que ce dernier prenne des mesures afin de réduire, par exemple, la consommation de tabac et la mortalité liée au cancer du poumon, les ministres se demandaient s'il n'était pas préférable une fois les faits connus de laisser la décision finale au jugement des individus ${ }^{75}$ pour éviter d'être accusé « d'attitude paternaliste sur la question $»^{76}$. Dans son arbitrage final, Enoch Powell prit en compte les considérations économiques et sanitaires ainsi que ses idées libérales sur le rôle de l'État et celui de l'individu dans la société ${ }^{77}$.

En avril 1959, le Royal College of Physicians, qui avait d'abord refusé cette initiative en novembre 1956 à cause de son ancien Président Lord Brain, décida de former une commission présidée par Robert Platt, dont la mission serait de préparer un rapport visant à établir le lien entre consommation de cigarettes et cancer des poumons. Cette commission publia ainsi un rapport le 6 mars $1962^{78}$, lequel confirmait bien que la cigarette provoquait ce cancer-là. Ce rapport soulignait en grande partie le rôle des médias et le consumérisme et préconisait la restriction, voire l'interdiction, de la publicité sur la vente de cigarettes après l'active contribution de Jerry Morris au sein de la commission, lequel avait montré que la publicité jouait un rôle indéniable dans l'augmentation des ventes de cigarettes ${ }^{79}$. Le Cabinet considérait la possibilité d'imposer des restrictions sur les publicités pour le tabac, mais reconnaissait que cela entrainerait de «sérieuses difficultés» dans sa mise en application ${ }^{80}$. Fallait-il recourir à la loi ${ }^{81}$, ou 
encourager les grandes firmes de l'industrie du tabac à limiter leurs spots publicitaires $?^{82}$ En dernier ressort, cela pourrait impliquer également que le gouvernement interdise ce genre de publicité dans la presse écrite ${ }^{83}$. Certaines grandes firmes de l'industrie du tabac décidèrent de limiter leurs spots publicitaires, en ne demandant plus de diffusion après $21 \mathrm{~h}$ et aux heures de grande audience par les enfants ${ }^{84}$. L'une d'elle, Imperial, estimait, d'une part, que cette initiative volontaire permettrait de réduire son budget publicité de $50 \%$ et que, de l'autre, elle éviterait au gouvernement d'imposer une interdiction totale ${ }^{85}$.

Après de nombreuses réunions et de multiples débats, Enoch Powell décida de rejeter tout projet de loi visant à interdire la publicité de ventes de cigarettes à la télévision ${ }^{86}$. Sa décision fut entérinée par le Cabinet le 26 novembre 1962, ${ }^{87}$ même s'il essuya de nombreuses critiques par la suite ${ }^{88}$. Plusieurs raisons peuvent expliquer son refus, en dépit des dangers que la cigarette représentait pour la santé. Tout d'abord, si une interdiction sur la publicité de ventes de cigarettes à la télévision ou dans la presse écrite avait été imposée, le gouvernement aurait ouvert la boite de Pandore. En effet, Enid Russell Smith, haut fonctionnaire au ministère de la Santé, pensait que, si ce rôle incombait au gouvernement, alors la situation ne s'arrêterait pas au problème de la cigarette. L'État devrait par conséquent légiférer sur tout ce qui pourrait nuire à la santé, comme l'alcool par exemple, et deviendrait ainsi un État gendarme ${ }^{89}$. Selon Godber qui ne partageait pas le point de vue du ministre de la Santé, Powell avait fait primer l'argument politique et libéral sur celui de la santé publique des individus. Il expliquait ainsi, se plaçant dans un contexte imaginaire où le tabac ne serait pas en vente libre, que si on devait autoriser la vente du tabac, il serait illogique d'en interdire la publicités ${ }^{0}$.

L'argument économique importait beaucoup à Powell qui ne voulait pas entrer en conflit avec le Trésor. La vente de cigarettes générait en effet des recettes non négligeables pour le gouvernement en raison de la TVA sur le tabac. Quelques années plus tard (en 1975), Powell revint sur les raisons qui l'avaient poussé à s'opposer à cette interdiction ${ }^{91}$. Il pensait que le gouvernement ne voulait pas avoir à réorganiser son système d'impôts indirects. Il serait tout aussi absurde pour un gouvernement de légiférer contre une forme de comportement commun et largement répandu: en soi, être fumeur ne représentait en rien un comportement marginal et dangereux pour autrui ${ }^{92}$. Powell avait donc appliqué ses croyances libérales à un domaine qui, pour l'époque, pouvait paraitre secondaire, si l'on considère que la commission sur la santé et le tabac envisageait bien d'autres solutions pour faire baisser la consommation de ce dernier, telles que son interdiction dans les lieux publics, l'augmentation des impôts indirects ou la prolifération des campagnes de prévention au sein des établissements scolaires ${ }^{93}$.

\section{La fluoration de l'eau : un libre choix?}

Ce fut avec un certain enthousiasme que le ministère de la Santé avait accueilli dans les années 1950 la proposition visant à fluorer l'eau, puisque cette mesure prophylactique, consistant simplement à ajouter du fluor dans l'eau potable, pouvait prévenir le risque carieux et ainsi permettre de réaliser des économies non négligeables sur le coût des traitements dentaires ${ }^{94}$. Ainsi, il nomma en 1956 une commission afin qu'elle détermine si la fluoration de l'eau ne présentait aucun danger pour la santé. La possibilité d'une telle initiative suscita une vive opposition de la part de différentes organisations, telles que la British Housewives League, la London Anti-Fluoridation Campaign et surtout la National Pure Water Association ${ }^{95}$, qui martelaient avec force que la municipalité devait fournir de 
l'eau «pure et saine $»^{96}$. Alors que le ministre de la Santé avait été chargé de préparer un projet de loi visant à autoriser les collectivités locales à procéder légalement à la fluoration de leur eau, après des signes favorables de l'Organisation Mondiale de la Santé et des études scientifiques encourageantes ${ }^{97}$, la Commission des affaires sociales choisit de reporter sa mise en place afin d'éviter une controverse avant les élections législatives. L'objectif financier de la mesure primait sur les avantages de santé publique, car il était clairement question pour les Conservateurs de diminuer le coût des traitements dentaires. Le problème à résoudre serait d'instaurer une loi lorsque des poursuites judiciaires seraient déjà lancées contre une collectivité locale ayant déjà ajouté illégalement du fluor dans ses réserves d'eau municipale ${ }^{98}$, comme celles d'Andover et de Watford qui avaient défrayé la chronique. De nombreuses questions importantes, laissées ainsi en suspens, allaient restreindre la marge de manœuvre de Powell. Conscient de ces enjeux, il affirma :

There is a good deal of popular misconception about ministers and policy decisions. I think a large section of the public imagines the competent minister bustling into his office on a Monday morning with a neatly written list of the new policies which he is going to put into force. In real life nearly all policy decisions emerge out of an existing situation. (...) This is even true in the extreme instance of a new government moving in after a decisive General Election in which they've defeated their opponents. They would still find most of the decisions they were taking had arisen out of a pre-existing situation ${ }^{99}$.

Enoch Powell hérita donc d'un dossier très controversé où plusieurs arguments étaient mis en avant pour condamner toute tentative d'imposer une fluoration obligatoire des eaux municipales. La motivation première du Cabinet était économique, dans une perspective de médecine préventive: elle permettrait de lutter contre la carie, alors considérée comme un véritable fléau, et ainsi d'éviter à moindre coût de futurs traitements dentaires. Charles Webster justifie le bien-fondé de cette initiative de l'État en ces mots: "The latter represented one of the simplest, most effective, and most economical measures capable of being applied in the field of preventive medicine $»^{100}$. Powell soutenait la mesure et affirma lors d'une réunion du Cabinet: "There was no doubt that the addition of fluoride to drinking water would greatly reduce decay in the teeth of children, that it would have no harmful side-effects »101.

En 1962, un rapport fut publié s'appuyant sur les conclusions univoques de trois enquêtes pilotes menées sur des eaux fluorées : la fluoration de l'eau avait permis de réduire le risque de caries chez les enfants sans aucun danger observé pour leur santé102. Ainsi, ces résultats scientifiques vinrent corroborer les dires de Powell, chargé de préparer un projet de loi. Puisque ce dernier était également très sensible à la question des libertés individuelles dans une société libérale, il imagina un plan ingénieux pour mettre en place la fluoration de l'eau, qui éviterait au gouvernement d'être accusé d'imposer une décision touchant directement toute la population. Il défendit son projet de loi à la Chambre des Communes en décembre 1962, qui consistait seulement en des mesures limitées visant à encourager la fluoration de l'eau. En s'appuyant sur l'article 28 de la loi sur le NHS de 1946, Powell offrait en effet la possibilité aux collectivités locales de soumettre une proposition à l'État visant à fluorer leur eau sous le régime existant du NHS, lequel les protégerait en cas de poursuites judiciaires éventuelles. Cette mesure s'apparentait en fait à une certaine forme de décentralisation, mais toujours sous l'égide du Cabinet qui en gardait le contrôle. Les résultats de cette politique furent cependant très limités, puisque, à court terme, seulement huit collectivités locales sur 1800 avaient soumis une demande 
au gouvernement en avril $1963^{103}$. À long terme, Charles Webster indique qu'en 1979, seulement $10 \%$ des eaux municipales contenaient du fluor ajouté, en raison d'une forte et constante opposition des lobbys ${ }^{104}$. Les conseillers politiques de Macmillan accusèrent Powell d'avoir très mal géré ce dossier.

Les lobbys s'opposèrent à la mesure de Powell en évoquant une raison éthique et morale, comme par exemple la National Pure Water Association ${ }^{105}$. Cette dernière contestait le bienfondé et la validité médicale de cette mesure et soulevait une question cruciale: quelle était la justification morale pour que chaque conseiller municipal oblige la population à ingurgiter une telle substance, potentiellement nocive qui plus est. Ce fut cet argument qui retint l'attention de Powell, qui laissa deux accolades à côté du point 5 de la circulaire :

Fluoridation infringes a fundamental human right which, in a free society, should be inalienable (viz: the right to decide, for ourselves, what substances intended to affect the development of our bodies shall go into our bodies) ${ }^{106}$.

Les individus pouvaient en effet librement décider de recourir à des comprimés de fluor après le brossage des dents ou ajouter du fluor en poudre pendant le brossage, comme le rappela en 1961 Sir Stanton Hicks, professeur émérite en physiologie humaine et pharmacologie de l'Université d'Adélaïde ${ }^{107}$. Selon A. E. Joll, la fluoration forcée des eaux sans le consentement des individus était assimilable à une pratique qui a toujours été perçue dans notre pays comme typique des régimes totalitaires ${ }^{108}$. Ce fut également la référence au régime soviétique, que Powell avait en horreur, en raison de la perte affichée des libertés individuelles dans un État communiste, qui retint son attention et qu'il marqua d'une double accolade dans la circulaire. Sa conception d'une société libérale avait été influencée par The Constitution of Liberty, écrit par F. V. Hayek en 1960. La cohérence du Powellisme allait être mise à mal dans plusieurs lettres privées que reçut Powell en 1964, alors qu'il venait d'affirmer que le Parti conservateur était le parti du libre choix, de la libre concurrence et de l'entreprise privée. L'une de ces missives souligne assez clairement la faille de sa vision :

When you were Minister of Health you recommended all local Authorities to add sodium fluoride to the drinking water and it was left to them to decide for or against fluoridation and not to the individual citizen. In view of this it certainly seems strange and out of place for you to speak of 'free choice' ${ }^{109}$.

Robert Shepherd note que Powell fut en définitive frustré de ne pas avoir réussi à régler cette question controversée et de ne pas avoir bien légiféré pour régler le problème ${ }^{110}$. Il avait opté pour une solution intermédiaire, qui ne produisit pas les effets économiques escomptés et qui allait fournir une arme à ses détracteurs, désireux de remettre en cause son projet libéral d'une société libérale tory.

\section{Conclusion}

Après son passage au ministère de la Santé, Powell laissa une empreinte durable tant il avait mis en œuvre différentes mesures visant à rationaliser, moderniser et humaniser le NHS. Manson estimait que son objectif global fut «de mettre une note de rationalité capitaliste dans le NHS ${ }^{111}$. Les actions de Powell révèlent un double impératif dans sa conception du NHS. Il pensait, d'une part, à l'intérêt collectif et il était donc primordial, à ses yeux, d'assurer aux patients l'égalité de l'accès aux soins dans des hôpitaux modernisés et humanisés. Il légitimait ainsi une ingérence étatique positive, puisque seul 
l'État pouvait décider de la construction d'un hôpital pour le bien collectif, à condition de responsabiliser ensuite les patients qui devaient prendre conscience que les ressources du NHS n'étaient pas illimitées. D'autre part, il défendait une vision publique du NHS tout en remettant le bénévolat au cœur du système et en encourageant dans une certaine mesure les canaux privés ${ }^{112}$. Son objectif d'humanisation du système public répondait aux exigences de la démarche disraélienne, qui avait fait de la santé publique un de ses grands objectifs en 1872.

Son expérience en tant que ministre de la Santé lui permit entre autres de confronter les sacrosaints principes de liberté individuelle et de choix avec le pouvoir puissant de la force étatique parfois nécessaire pour améliorer une situation. T. E. Utley ${ }^{113}$ avait toutefois rencontré à l'époque des difficultés à rendre compte de l'adéquation entre les actions de Powell à ce ministère et sa philosophie libérale qui se dessinait sous l'étiquette du Powellisme :

The task was to administer this service as efficiently as possible. It would continue to exhibit the defects of a monopoly. (...) Powell has never wholly expelled from his mind the proposition that, if life is not to be controlled by the interplay of market forces, it will be better controlled by strong than by weak government ${ }^{114}$.

Cet article a tenté de rendre compte, par le biais de l'étude de l'industrie pharmaceutique, de la publicité sur le tabac et la fluoration de l'eau, des choix de Powell, toujours influencé par l'œuvre libérale de F. V. Hayek - le chantre du libre choix et de la liberté individuelle.

Par ailleurs, Powell fut la cible de critiques libérales, qui lui reprochèrent de ne pas avoir assez développé le système privé de santé. Il ne parvint pas en effet à garantir la gratuité des médicaments pour les patients du secteur privé, qui ne furent pas encouragés à aller consulter des médecins hors du cadre du NHS. Ainsi, Rudolf Klein écrit: «Denied the opportunity to exit from a service with a near-monopoly of employment for health care professionals, they engaged in voice in the NHS, i.e. the politics of protest ${ }^{115}$. Cependant, le Parti conservateur semble avoir voulu protéger un NHS public et intervenir dans le domaine de la santé publique jusqu'à la fin des années 1980. Quant à l'opinion publique, elle a toujours été encline à soutenir un système de soins gratuits, peu importe les orientations politiques. Ce climat rendait donc compliqué un changement de philosophie avant les années Thatcher.

À la suite du passage de Powell au ministère de la Santé, les années 1960 furent aussi marquées par un autre débat sur la nature du NHS ${ }^{116}$ qui se posait en ces termes: « Was the problem one of constraining the demands made by consumers of health care or of restraining the demand generated by the producers of health care? ». Ce questionnement allait de nouveau être l'occasion pour une confrontation entre les théoriciens des forces du marché et les défenseurs du système public de santé. Powell avait déjà clairement mis le doigt sur ce problème de manière pessimiste : il semblait impossible de restreindre la demande des usagers. Richard Crossman, secrétaire d'État travailliste des services sociaux en 1969, formulait le même diagnostic pessimiste, légitimant quelque peu la thèse des théoriciens du secteur privé: "The trouble is that there is no foreseeable limit on the social services which the nation can reasonably require except the limit that the Government imposes $»^{117}$.

Stéphane Porion, agrégé d'anglais, Maitre de Conférences en civilisation britannique à l'Université de Tours, a rédigé son doctorat sur les idées politiques et économiques d'Enoch Powell de 1946 à 1968. Il a beaucoup publié sur cet homme politique et récemment codirigé un ouvrage avec Olivier Esteves, publié chez 
Routledge en mai 2019 et intitulé The Lives and Afterlives of Enoch Powell: The Undying Political Animal. Ses travaux portent également sur le Parti conservateur britannique depuis 1945, ainsi que sur les droites radicales depuis 1967.

\section{BIBLIOGRAPHIE}

Sources primaires

Archives

National Archives (London)

- Prime Minister's Office records

NA, PRO PREM 11/3741, PREM 11/5203, PREM 11/3438

- Treasury records

NA, PRO, T227/1383

- Cabinet and its Committees records

NA, PRO CAB 129/104, C(61), 155, CAB 134/1976, CAB 128/36, CC(62)19, CAB 128/36, CC(62)46, CAB 130/185, GEN.762/1, CAB 130/185, GEN.763/1, CAB 128/36, CC(62)19, CAB 130/185, GEN.763/13, CAB 21/4878, CAB 130/185, GEN.763/16, CAB 134/1972, CAB 134/1974, CAB 134/1975, CAB $128 / 36, \mathrm{CC}(62) 73$,

- Public Health

NA, PRO MH 55/2227, PRO MH 55/2204,

Churchill Archives Centre (Cambridge)

POLL 4/1/1, File 5, POLL 4/1/27, File 1, POLL 1/1/13, POLL 4/1/1, File 6, POLL 3/1/30, Powell Papers.

London School of Economics Archives (London)

BOAPAH, 1980, LSE Library Archives and Special Collections.

Publiées

Aneurin Bevan, In Place of Fear (Londres, Quartet, 1978).

Rex Collings (ed.), Reflections of a Statesman (Londres, Bellew, 1991).

Enoch Powell, A New Look at Medicine and Politics (Londres, Pitman Medical, 1966).

Enoch Powell, 'The Limits to Laissez-Faire', Crossbow, vol.3 no.11, printemps 1960, pp. 25-28.

Enoch Powell, 'My Years as Health Minister', The Spectator, 20 février 1988, pp. 8-10.

John Wood (ed.), A Nation Not Afraid (Londres, Batsford, 1965).

Sources secondaires

Nicholas Barr, The Economics of the Welfare State (Oxford, OUP, 1993). 
Virginia Berridge, Health and Society in Britain since 1939 (Cambridge, CUP, 1999).

V. Berridge, 'Medecine and the Public: the 1962 Report of the Royal College of Physicians and the New Public Health', Bulletin of the History of Medecine, 2007 Spring; 81(1), pp. 286-311,

http://www.pubmedcentral.nih.gov/articlerender.fcgi?artid=1894742 [Dernière consultation le 17 juillet 2008].

Paul Bridgen \& Rodney Lowe, Welfare Policy Under the Conservatives (1951-1964) (Kew, London Public Record Office Publications, 1998).

Tony Cutler, 'Economic Liberal or Arch Planner? Enoch Powell and the Political Economy of the Hospital Plan', Contemporary British History, vol. 25, n 4, 2011, pp. 469-489.

Howard Glennerster, British Social Policy since 1945 (Oxford, Blackwell, 2000).

Simon Heffer, Like the Roman: The Life of Enoch Powell (Londres, Phoenix, 1999).

Ian Holliday, The NHS Transformed (Manchester, Baseline Book Company, 1995).

Norman Hunt (ed.), WhiteHall and Beyond, Jo Grimmond, Enoch Powell, Harold Wilson (Three

Conversations with Norman Hunt) (Londres, BBC, 1964).

Brian Inglis, Drugs, Doctors and Disease (Londres, André Deutsch, 1965).

Rudolf Klein, The New Politics of the NHS (Harlow, Pearson Education, 2001).

Rodney Lowe, The Welfare State in Britain since 1945 (Basingstoke: Macmillan, 1999).

John Mohan, Planning, Markets and Hospitals (Londres, Routledge, 2002)

John Mohan, "Milburn, Powell and Hayek: For and Against Planning in the NHS", Journal of Health Services Research and Policy, 9 (1), 2004, pp. 54-56.

Stéphane Porion, « 'A Local Difficulty'? Enoch Powell's Secret Motivation to Resign from the Treasury in 1958 and its Aftermath ", in Fabienne Portier- Le Cocq, Secret and Lies in the United Kingdom: Analysis of Political Corruption (Washington DC, Westphalia Press, 2017), pp. 47-73.

Timothy Raison, Tories and the Welfare State: A History of Conservative Social Policy since the Second World War (Basingstoke, Macmillan, 1990).

G. Rivett, From Cradle to Grave, Fifty Years of the NHS (Londres, King's Fund, 1998).

Robert Shepherd, Enoch Powell: A Biography (Londres, Pimlico, 1997).

Neil Small, Politics and Planning in the NHS (Miltonkeynes, Open University Press, 1989).

N. Timmins, The Five Giants, A Biography of the Welfare State (Londres, William Collins, 2017).

T. E. Utley, Enoch Powell: The Man and his Thinking (Londres, William Kimber, 1968).

Brian Watkin, The National Health Service: The First Phase 1948-1974 and After (Londres, George Allen \& Unwin, 1978).

Charles Webster, 'Conservatives and Consensus: The Politics of the National Health Service, 1951-1964', in Ann Oakley \& A. Susan Williams (eds.), The Politics of the Welfare State (Londres, UCL, 1994), pp. 54-74.

Charles Webster, The National Health Service: A Political History (Oxford, OUP, 2002).

Presse spécialisée

British Medical Journal 
J. Seale, 'The Health Service in an Affluent Society', British Medical Journal, 1er septembre 1962, pp. 598-602.

'The Minister of Health', British Medical Journal, 26 octobre 1963, p. 1012.

'Is there an Alternative? Medicine and Politics', British Medical Journal, 4 mars 1967,

pp. 555-559.

Webographie

http://www.econ.yale.edu/ nordhaus/homepage/homepage/PASandGPG.pdf (site consulté le 2 février 2019).

https://aheblog.com/2017/03/07/kenneth-arrow-on-healthcare-economics-a-21st-centuryappreciation/ (site consulté le 2 février 2019).

\section{NOTES}

1. Aneurin Bevan, In Place of Fear (Londres, Quartet, 1978), p. 109.

2. Ibid., p. 100.

3. Rodney Lowe, The Welfare State in Britain since 1945 (Basingstoke: Macmillan, 1999), p. 195.

4. Charles Webster, 'Conservatives and Consensus: The Politics of the National Health Service, 1951-1964', in Ann Oakley \& A. Susan Williams (eds.), The Politics of the Welfare State (Londres, UCL, 1994), p. 56; Rudolf Klein, The New Politics of the NHS (Harlow, Pearson Education, 2001), p. 51.

5. Paul Bridgen \& Rodney Lowe, Welfare Policy under the Conservatives (1951-1964) (Kew, London Public Record Office Publications, 1998), p. 62; Rodney Lowe, The Welfare State in Britain since 1945, p. 187; Howard Glennerster, British Social Policy since 1945 (Oxford, Blackwell, 2000), p. 81.

6. Nicholas Timmins, The Five Giants: A Biography of the Welfare State (Londres, Harper Collins, 2017), p. 208.

7. Enoch Powell, A New Look at Medicine and Politics (Londres, Pitman Medical, 1966), pp. 6-7.

8. Ibid., pp. 14-15. Voir également NA, PRO PREM 11/3741, B. Trend minute, 22 décembre 1958.

9. Robert Shepherd, Enoch Powell: A Biography (Londres, Pimlico, 1997), p. 210.

10. Stéphane Porion, «'A Local Difficulty'? Enoch Powell's Secret Motivation to Resign from the Treasury in 1958 and its Aftermath », in Fabienne Portier- Le Cocq, Secret and Lies in the United Kingdom: Analysis of Political Corruption (Washington DC, Westphalia Press, 2017), pp. 47-73.

11. Charles Webster, 'Conservatives and Consensus', p. 63.

12. Enoch Powell, A New Look at Medicine and Politics, p. 2.

13. Enoch Powell, Discours au Congrès annuel du Parti conservateur, 7 octobre 1963, p. 61, POLL 4/1/1, File 5, Powell Papers.

14. Enoch Powell, 'Humanisation of Hospital Service - Minister of Health Review', 19 octobre 1962, pp. 1-5, POLL 4/1/1, File 5, Powell Papers.

15. Robert Shepherd, Enoch Powell: A Biography, pp. 204-243; S. Heffer, Like the Roman: The Life of Enoch Powell (Londres, Phoenix, 1999), pp. 266-318.

16. Ian Holliday, The NHS Transformed (Manchester, Baseline Book Company, 1995), p. 9.

17. Brian Watkin, The National Health Service: The First Phase 1948-1974 and After (Londres, George Allen \& Unwin, 1978), pp. 118-119.

18. Virginia Berridge, Health and Society in Britain since 1939 (Cambridge, CUP, 1999), p. 26.

19. Charles Webster, The National Health Service: A Political History (Oxford, OUP, 2002), p. 36.

20. Tony Cutler, 'Economic Liberal or Arch Planner? Enoch Powell and the Political Economy of the Hospital Plan', Contemporary British History, vol. 25, n 4, 2011, pp. 469-489.

21. Paul Bridgen \& Rodney Lowe, Welfare Policy under the Conservatives (1951-1964), pp. 56-61. 
22. Brendon Sewill cité dans Timothy Raison, Tories and the Welfare State: A History of Conservative Social Policy since the Second World War (Basingstoke, Macmillan, 1990), p. 48.

23. Ibid., p. 49.

24. Ibid., p. 50.

25. Ibid., pp. 50 \& 51 .

26. Charles Webster, The National Health Service: A Political History (Oxford, OUP, 2002), p. 36.

27. Ibid.

28. Enoch Powell, A New Look at Medicine and Politics, p. 68.

29. Enoch Powell, 'The Limits to Laissez-Faire', Crossbow, vol.3 no.11, printemps 1960, p. 25.

30. Ibid., p. 26.

31. Voir par exemple: http://www.econ.yale.edu/ nordhaus/homepage/homepage/ PASandGPG.pdf (site consulté le 2 février 2019).

32. Voir par exemple: https://aheblog.com/2017/03/07/kenneth-arrow-on-healthcareeconomics-a-21st-century-appreciation/ (site consulté le 2 février 2019).

33. Ibid.

34. Ibid.

35. Ibid.

36. BOAPAH, 1980, p. 15, LSE Library Archives and Special Collections.

37. Transcription du débat entre Powell et le Dr. Henry Miller, « A New Look at Medicine », Third Programme, 1er décembre 1966, p. 2, POLL 4/1/27, File 1, Powell Papers; 'Is there an Alternative? Medicine and Politics', British Medical Journal, 4 mars 1967, pp. 555-559.

38. Ibid., p. 14.

39. Ibid., p. 16.

40. Nicholas Barr, The Economics of the Welfare State (Oxford, OUP, 1993), p. 433.

41. Enoch Powell, 'Social Services', The Times Health Supplement, 13 novembre 1981, in Rex Collings (ed.), Reflections of a Statesman (Londres, Bellew, 1991), p. 582.

42. Enoch Powell, 'My Years as Health Minister', The Spectator, 20 février 1988, p. 9.

43. Ibid.

44. Ibid.

45. Neil Small, Politics and Planning in the NHS (Miltonkeynes, Open University Press, 1989), p. 11.

46. Enoch Powell et Dr. Henry Miller, 'A New Look at Medecine', pp. 2, 3 \& 16.

47. ' 'The Minister of Health', British Medical Journal, 26 octobre 1963, p. 1012; N. Timmins, The Five Giants, A Biography of the Welfare State (Londres, William Collins, 2017), p. 208; G. Rivett, From Cradle to Grave, Fifty Years of the NHS (Londres, King's Fund, 1998), p. 132.

48. Enoch Powell, A New Look at Medicine and Politics, p. 14.

49. Ibid., p. 16.

50. Robert Shepherd, Enoch Powell: A Biography, p. 218. Voir aussi NA, PRO, T227/1383 et Enoch Powell et Dr. Henry Miller, 'A New Look at Medecine', pp. 15-16.

51. Powell changea d'avis quant à l'efficacité de mettre en place le PESC en 1961. Il écrivit d'abord un plaidoyer contre cette idée le 16 septembre 1961, puis changea vraisemblablement d'avis en octobre 1961, après avoir reçu une réponse du Trésor le 27 septembre 1961. POLL 1/1/13, Powell Papers.

52. Powell rédigea par exemple une note pour le Premier Ministre, dans laquelle il montra sa détermination à contenir les revendications de hausse salariale des infirmières selon l'objectif de 2,5\% fixé par le Trésor. Lettre de Powell à Macmillan, 10 avril 1962, §8, p.3, NA, PRO PREM 11/5203.

53. Enoch Powell, A New Look at Medicine and Politics, p. 73.

54. NA, PRO PREM 11/3438, lettre de Macmillan à Powell, $1^{\text {er }}$ août 1960.

55. Robert Shepherd, Enoch Powell: A Biography, p. 235. 
56. Enoch Powell, 'Cost of NHS Drugs - Health Minister on Relations with Industry', 26 avril 1961, p. 1, POLL 4/1/1, File 6, Powell Papers.

57. Brian Inglis, Drugs, Doctors and Disease (Londres, André Deutsch, 1965), pp. 11-12.

58. Voir NA, PRO CAB 129/104, C(61), 155, 'National Health Service : The Drug Bill - Memorandum by the Minister of Health', 6 octobre 1961.

59. Enoch Powell, A New Look at Medicine and Politics, p. 64.

60. Brian Inglis, Drugs, Doctors and Disease, p. 9.

61. Medical News, 8 mai 1964, in ibid., p. 30.

62. Ibid.

63. Ibid., p. 199.

64. Enoch Powell, A New Look at Politics and Medicine, p. 64.

65. Enoch Powell, 'Cost of NHS Drugs - Health Minister on Relations with Industry', p. 1.

66. Ibid., p. 57.

67. Enoch Powell, A New Look at Politics and Medicine, pp. 65-66.

68. Enoch Powell confia plus tard qu'il n'avait pas ressenti le besoin de se faire aider par un homme d'affaires et avait mené les négociations seul, car cette décision relevait de la responsabilité et de la transparence publiques vis-à-vis du Parlement et non des compétences du privé. Voir Norman Hunt (ed.), WhiteHall and Beyond, Jo Grimmond, Enoch Powell, Harold Wilson (Three Conversations with Norman Hunt) (Londres, BBC, 1964), p. 55.

69. The Times, 18 mai 1961, in Robert Shepherd, Enoch Powell: A Biography, p. 236.

70. Brian Inglis, Drugs, Doctors and Disease, p. 9.

71. Ibid., pp. 10-11; Enoch Powell, A New Look at Politics and Medicine, p. 66.

72. Ibid.

73. NA, PRO CAB 134/1976, '5e reunion du Home Affairs Committee', 20 mars 1959, p. 7.

74. V. Berridge, 'Medecine and the Public: the 1962 Report of the Royal College of Physicians and the New Public Health', Bulletin of the History of Medecine, 2007 Spring; 81(1), p. 2.

http://www.pubmedcentral.nih.gov/articlerender.fcgi?artid=1894742 [Dernière consultation le 17 juillet 2008].

75. NA, PRO CAB 128/36, CC(62)19, 6 mars 1962, p. 6.

76. NA, PRO CAB 128/36, CC(62)46, 12 juillet 1962, p. 3.

77. Voir par example, John Mohan, "Milburn, Powell and Hayek: For and Against Planning in the NHS", Journal of Health Services Research and Policy, 9 (1), 2004, pp. 54-56.

78. NA, PRO CAB 130/185, GEN.762/1, 'Réunion du comité sur la santé et le tabac', 28 février 1962, p. 1.

79. V. Berridge, 'Medecine and the Public', pp. 7-8.

80. NA, PRO CAB 130/185, GEN.762/1, 'Réunion du comité sur la santé et le tabac', p. 1.

81. NA, PRO CAB 130/185, GEN.763/1, 'Réunion du comité sur la santé et le tabac', 23 mars 1962, p. 3; NA, PRO CAB 128/36, CC(62)19, 6 mars 1962, p. 5.

82. NA, PRO CAB 130/185, GEN.763/13, 'Réunion du comité sur la santé et le tabac', 15 mai 1962, p. 9.

83. Ibid.

84. Ibid; NA, PRO CAB 21/4878, Lettre du Lord President au Premier Ministre, 25 juillet 1962.

85. V. Berridge, 'Medecine and the Public', p. 12.

86. NA, PRO MH 55/2227, 'Note de service d'Enoch Powell', 11 novembre 1962.

87. NA, PRO CAB 130/185, GEN.763/16, 'Note de service du ministre de la Santé', 26 novembre 1962, p. 1.

88. Robert Shepherd, Enoch Powell: A Biography, p. 233.

89. NA, PRO MH 55/2204, 'Note de service d'Enid Russell Smith', 5 février 1962. Ce point de vue est corroboré par toute la commission sur la santé et le tabac. Voir NA, PRO CAB 130/185, GEN.763/13, 'Réunion du comité sur la santé et le tabac', 15 mai 1962, p. 9. 
90. Godber cité dans Simon Heffer, Like the Roman, p.293. Godber ajouta que ce fut le gouvernement travailliste de Wilson qui interdit les publicités de ventes de cigarettes à la télévision en 1965.

91. V Berridge, 'Medecine and the Public', p. 13.

92. Il faut considérer qu'à l'époque, l'effet nocif du tabagisme passif n'était pas d'actualité.

93. NA, PRO CAB 130/185, GEN.763/13, 'Réunion du comité sur la santé et le tabac', 15 mai 1962, pp. 9-13.

94. Voir NA, PRO CAB 134/1972, CAB 134/1974 et CAB 134/1975.

95. Ibid.

96. NA, PRO CAB 128/36, CC(62)73, 6 décembre 1962, p. 11.

97. Il est précisé dans la $19^{\mathrm{e}}$ réunion du Home Affairs Committee que les résultats scientifiques de cet ajout ne seraient sûrement publiés qu'en 1961. Voir NA, PRO CAB 134/1976, '19 réunion du Home Affairs Committee', 6 novembre 1959, p. 8.

98. Voir Ibid et NA, PRO CAB 134/1976, '11 e réunion du Home Affairs Committee', 3 juillet 1959, p. 3.

99. Norman Hunt (ed.), Whitehall and Beyond, p. 45.

100. Charles Webster, The National Health Service, A Political History, p. 129.

101. NA, PRO CAB 128/36, CC (62) 73, p. 11.

102. Ministry of Health, The Conduct of the Fluoridation Studies in the UK and the Results Achieved after Five Years (Londres, HMSO, 1962).

103. Robert Shepherd, Enoch Powell: A Biography, p. 232.

104. Charles Webster, The National Health Service, A Political History, p. 129.

105. The National Pure Water Association, 'Fluoridation of Public Water Supplies, The Primary Question of Principal', [n.d.], POLL 3/1/30, Powell Papers.

106. Ibid.

107. Sir Stanton Hicks cité dans A. E. Joll, Lettre à l'éditeur, reproduite dans The Lancet, 8 février 1964, p. 326, POLL 3/1/30, Powell Papers.

108. Ibid.

109. Lettre de E. T. Forrest (Southampton) à Enoch Powell, 6 avril 1964, POLL 3/1/30, Powell Papers.

110. Robert Shepherd, Enoch Powell: A Biography, p. 232.

111. Manson cité dans John Mohan, Planning, Markets and Hospitals (Londres, Routledge, 2002) p. 111.

112. E. Powell, 'Discours à Crewkerne', 20 septembre 1963, in John Wood (ed.), A Nation Not Afraid (Londres, Batsford, 1965), p. 44.

113. Ce fut le premier à écrire un ouvrage sur le Powellisme en 1968.

114. T. E. Utley, Enoch Powell:The Man and his Thinking (Londres, William Kimber, 1968) pp. 78-79.

115. Rudolf Klein, The New Politics of the NHS, p. 53.

116. British Medical Journal, 26 novembre 1966, pp. 1315-1319; J. Seale, 'The Health Service in an Affluent Society', British Medical Journal, $1^{\mathrm{er}}$ septembre 1962, pp. 598-602.

117. R Crossman, Paying for the Social Services (Londres, Fabian Society, 1969), in Rudolf Klein, The New Politics of the NHS, p. 55. 


\section{RÉSUMÉS}

Enoch Powell fut nommé ministre de la Santé en juillet 1960 par Harold Macmillan, puis élevé au rang de ministre du Cabinet en 1962. Calcul politique de la part du Premier ministre, qui ne portait pas Powell dans son cœur, visant à la fois à l'empêcher de critiquer le gouvernement avec son credo libéral pendant les années de la mise en œuvre de la Middle Way et à le mettre en difficulté à la tête d'un ministère assez dispendieux. Powell tenta d'imprimer sa marque et de rompre avec les politiques de ses prédécesseurs : il s'agissait pour lui de rationaliser les dépenses de son ministère, tout en suivant un objectif de modernisation et d'humanisation du NHS par le biais de la mise en place d'un plan ambitieux de construction de nouveaux hôpitaux.

Powell était convaincu que le NHS pouvait être modernisé et défendit une vision publique de ce dernier tout en encourageant les canaux privés. Cet article a pour objectif d'analyser l'approche iconoclaste de Powell en s'appuyant sur les archives nationales de Londres et celles de Powell détenues à Cambridge. Afin de compléter les analyses historiographiques, l'étude se penchera sur trois points (gestion powellienne des questions du coût des médicaments, de la publicité sur le tabac et de la fluoration de l'eau) afin de démontrer comment Powell dut gérer un autre dilemme que celui d'humaniser et rationnaliser en même temps le NHS : jusqu'à quel point était-il possible de défendre les idées de liberté et de choix dans un système public de santé où la puissance étatique était force motrice?

Enoch Powell was appointed Minister of Health by Harold Macmillan in July 1960, before being promoted to the position of Cabinet Minister in 1962. This was seen as a political manoeuvre from a Prime Minister who was hardly well disposed towards Powell. He thus planned to both prevent him from attacking the government with his free market beliefs in the years of the implementation of the Middle Way and put him in a difficult position at the head of a costly department. Powell attempted to leave his mark on it and break with his predecessors' policies: he intended to streamline NHS spending, while at the same time modernising and humanising the NHS through the introduction of an ambitious Hospital Plan. Powell was convinced that the NHS could be modernised. He believed that the latter should remain in the public domain but at the same time supported private health investment. This paper analyses Powell's iconoclastic approach by using primary sources from Kew's national archives and the Powell Papers from Cambridge. In order to enhance historiographical debates, the analysis will focus on three particular points: Powell's handling of the cost of drugs, cigarette advertising and the fluoridation of water. These issues reveal an additional dilemma that Powell had to face: to what extent could freedom of choice be introduced into a public health service in which the State was the main driver?

\section{INDEX}

Mots-clés : industrie pharmaceutique, publicité sur le tabac, Hayek, Disraeli, hôpitaux, fluoration de l'eau

Keywords : pharmaceutical industry, cigarette advertising, Hayek, Disraeli, hospitals, fluoridation of water 
AUTEUR

STÉPHANE PORION

Université de Tours 